\title{
Modelo metodológico para la educación ambiental a partir del museo de ciencias
}

\section{Methodological model for environmental education from the science museum}

\author{
SÁNCHEZ-RAMOS, Ma. Eugenia†* \& ZÁRATE-NEGRETE, Laura Elena \\ Universidad de Guanajuato, División de Ciencias Económico Administrativas \\ ID 1 ${ }^{\mathrm{er}}$ Autor: Ma. Eugenia, Sánchez-Ramos / ORC ID: 0000-0002-9277-0808, CVU CONACYT ID: 236588 \\ ID $1^{\mathrm{er}}$ Coautor: Laura Elena, Zárate-Negrete / ORC ID: 0000-0001-7520-8314, CVU CONACYT ID: 744432
}

DOI: $10.35429 / J C P .2019 .7 .3 .1 .7$

Recibido 13 de Enero, 2019; Aceptado 30 de Marzo, 2019

\section{Resumen}

Objetivo: El objetivo de la investigación es conocer los beneficios que ofrece el Museo de Ciencias en la educación ambiental en el nivel básico educativo y cómo integrarse a través de talleres al currículo formal. Metodología: En el presente trabajo de revisión documental se exponen los beneficios del aprendizaje a través de los museos y como potencializan el conocimiento adquirido en la población infantil en la educación ambiental. Se presenta un modelo metodológico sustentado en tres ejes primordiales para la educación ambiental: Contextualización, Sensibilización y Acción. Contribución: La educación formal, por tanto, debe contemplar espacios de aprendizaje externos para reforzar y/o ampliar el conocimiento. En este sentido, la divulgación de la ciencia toma un papel importante ya que es a través de actividades externas al programa académico donde lo aprendido en las aulas toma sentido en la vida cotidiana y situaciones prácticas. Lo anterior, hace necesario contar con nuevas estrategias educativas para entender y buscar diferentes soluciones que contrarresten el deterioro ambiental de nuestra época, utilizando la educación ambiental como una herramienta básica para que las personas adquieran conciencia de la importancia de preservar el medio en el que se encuentran y sean capaces de generar cambios en su conducta, valores, y estilos de vida.

Educación, Divulgación, Museos

\begin{abstract}
Resumen
The objective of the research is to know the benefits offered by the Science Museum in environmental education at the basic educational level and how to integrate through workshops into the formal curriculum. Methodology: In the present documentary review work, the benefits of learning through museums and how they potentiate the knowledge acquired in the child population in environmental education are exposed. A methodological model based on three main axes for environmental education is presented: Contextualization, Awareness and Action. Contribution: Formal education, therefore, must contemplate external learning spaces to reinforce and / or expand knowledge. In this sense, the dissemination of science takes an important role since it is through activities outside the academic program where what is learned in the classroom takes on meaning in everyday life and practical situations. The foregoing makes it necessary to have new educational strategies to understand and seek different solutions that counteract environmental deterioration of our time, using environmental education as a basic tool for people to become aware of the importance of preserving the environment in which they live. find and be able to generate changes in their behavior, values, and lifestyles.
\end{abstract}

Education, Outreach, Museums

Citación: SÁNCHEZ-RAMOS, Ma. Eugenia \& ZÁRATE-NEGRETE, Laura Elena. Modelo metodológico para la educación ambiental a partir del museo de ciencias. Revista de Pedagogía Crítica. 2019, 3-7: 1-7

\footnotetext{
* Correspondencia al Autor (Correo electrónico: sanchez.me@ugto.mx)

$\dagger$ Investigador contribuyendo como primer autor.
} 


\section{Introducción}

Hoy en día los museos han dejado de ser bodegas de almacenamiento de bienes preciosos y patrimonio histórico, para convertirse en espacios de divulgación que fortalecen al aprendizaje formal. Esta evolución, menciona Suárez (2005, en Álvarez, 2009) es consecuencia de varios sucesos:

a. los museos se han convertido en instituciones culturales, debido a la exigencia de la sociedad y de los medios,

b. La educación forma parte de las funciones del museo,

c. El museo mediatiza sus actividades, compitiendo con los medios de comunicación,

d. la tecnología ha permitido que los usuarios virtuales tengan acceso a ellos;

e. Los museos son entidades sustentables, ya que requieren de la generación de recursos que les permitan su existencia.

En el modelo educativo, la educación es un proceso permanente en el ser humano, es decir educar no se limita a la asistencia a una institución educativa y aprender en un determinado periodo de tiempo una serie de contenidos y destrezas sociales considerados suficientes para toda la vida (Álvarez, 2009). En base a lo anterior, los espacios externos a las instituciones oficiales de educación, como el museo en todas sus tipologías pueden ser alternativas de reforzamiento del aprendizaje, a través de actividades relacionadas con el plan de estudios de los diversos niveles educativos lo cual involucra reflexión y poner en práctica el conocimiento aprendido.

El documento se centra en diseñar un modelo metodológico que permita a la educación formal en el nivel básico una herramienta para la educación ambiental, sustentado en tres ejes primordiales: contextualización, sensibilización y acción. El artículo se encuentra estructurado de la siguiente forma: en un inicio se muestra el sustento epistemológico en el marco conceptual referente a la educación y el rol del museo de ciencias en la educación. En la parte metodológica se propone un modelo para la educación ambiental para el nivel básico debido a que la temática ambiental requiere de refuerzo del conocimiento del entorno, así como del sistema valoral que conlleve a la reflexión y cambio de actitudes y comportamiento.

ISSN 2523-2479

ECORFAN $^{\circledR}$ Todos los derechos reservados
La metodología implementada es el estudio de caso. Finalmente, los resultados exponen la estructura del modelo integrando actividades y los recursos existentes en un museo, así como las conclusiones derivadas de la investigación.

\section{Marco Conceptual}

\section{Educación formal, no formal e informal}

A continuación, se diferencia los tipos de educación existente para una mejor comprensión de la vinculación del museo con las instituciones educativas. La educación formal se caracteriza por ser estructurada, institucionalizada, y otorga grados académicos que siguen un orden ascendente; mientras que la educación no formal como lo es la divulgación son actividades organizadas y sistematizadas que se ofrecen para reforzar el aprendizaje formal pero no confieren ningún título o grado oficial; y finalmente la educación informal es la que se obtiene del entorno.

Otro aspecto que dificulta la vinculación entre los tipos de educación se debe a que los objetivos de aprendizaje de los espacios no formales difieren entre ellas, mientras que las escuelas se enfocan en el aprendizaje de contenidos conceptuales los espacios no formales enfatizan el interés, motivación, emoción y relaciones entre los visitantes y la ciencia para reforzar el conocimiento (Fenichel y Schweingruber, 2010).

Teniendo entendido los tipos de educación, los museos consideran esta nueva modalidad: institución museístico-pedagógica (Álvarez, 2009), en la cual participa directamente con la educación a través de materiales, exposiciones, charlas, cursos, talleres orientados a grupos escolares organizados; lo cuales ofrecen la ventaja de usuarios colectivos que aprovechen las instalaciones y compartan el conocimiento que el museo ofrece.

Los sistemas educativos formal, no formal e informal, son complementarios y se refuerzan entre sí. Ninguno de ellos presenta una superioridad de rango o eficacia sobre los demás, de tal forma que son los tres juntos los que conforman una red de aprendizajes, que posibilita el de todos los miembros de la sociedad. (González, 2001) 


\section{El museo de ciencias y su impacto en la educación formal}

Domínguez (2003) hace una reflexión del rol del museo actualmente, para él ha trascendido a espacios donde se descubre el conocimiento, pero también ofrece la posibilidad de ser un punto de reunión y de interacciones sociales que potencializa el pensamiento crítico y la concientización de los individuos. Otros autores reconocen que son lugares de memoria, como lo menciona Hernández (2013) “donde los recuerdos del pasado se convierten en una cuestión crítica del presente, en material para el ejercicio colectivo de la reflexión, lugares con ideología y posicionamientos que se convierten en medios de comunicación y difusión que promueven reflexiones" (citado en Megar, 2019, p.171).

La visión de institución museísticopedagógica término que utiliza Álvarez Domínguez (2009), permite que las visitas a los museos se conviertan no sólo en créditos para los programas educativos o actividades extracurriculares, que en muchas ocasiones no tienen impacto en el aprendizaje ya que sólo exigen la contemplación de alguna exposición que no tiene relación con el currículo; sino en una herramienta de profundización en el conocimiento que se obtiene de la escuela siendo una secuencia de este.

El aprendizaje en contextos no formales se orienta por la curiosidad y se mantiene por el afán de superar los retos que se plantean al interaccionar con el entorno (Guisasola, \& Morentin, 2007). Es por esto, que es importante que, para cumplir con el refuerzo del aprendizaje, se vincule al material a exponer los contenidos, actitudes y comportamientos de los visitantes. En este proceso, en conjunto con la escuela y previo a la visita se selecciona los temas para posteriormente definir las estrategias de divulgación idóneas de acuerdo a la edad y el nivel educativo de los usuarios que puede variar desde la experiencia vivencial hasta la simulación virtual.

Cabral \& Maldonado (2019) resaltan que el conocimiento científico al incrementar el los individuos el nivel cultural y educativo impacta en la reflexión, opinión y comprensión del entorno; por lo que los efectos son positivos y medibles a mediano y largo plazo.
Los museos de ciencias, que es el caso particular de este trabajo, tienen su origen en los gabinetes de ciencias en el siglo XVIII. Constituyen un medio para el conocimiento del patrimonio natural que es la base de la investigación sobre la tierra y la vida y que incide en la comprensión del mundo natural y de los seres vivos (Omedes, 2005).

\section{Metodología}

Resulta de gran importancia señalar que el conocimiento significativo requiere de un contexto real no imaginario, en donde inciden las experiencias sociales, personales y físicas. Es aquí donde el museo adquiere importancia como espacio ya que permite a cada individuo profundizar en el conocimiento de forma autónoma, colectiva o individual.

Como ya se ha mencionado, es fundamental el asociar las experiencias con los contenidos educativos con la finalidad de evitar temas aislados que no impacten en el refuerzo del conocimiento adquirido en las aulas.

Las teorías actuales que sustentan los programas de estudio en México son el constructivismo y el aprendizaje por competencias. El constructivismo establece que el individuo construye su propio aprendizaje mientras aprende, es decir, la interacción con el entorno genera nuevos conocimientos y puede ser individual o colectivo. El aprendizaje por competencias se enfoca al desarrollo individual de conocimientos y habilidades. (Hein, 2012).

En base a lo anterior, el usuario es responsable de la construcción del conocimiento personal en base a la exhibición o a la estrategia de divulgación. Ahora bien, el museo requiere de un público específico para situar tanto el conocimiento como la estrategia de aprendizaje. Caso de estudio: La educación ambiental en educandos del nivel educativo básico en el Museo de Historia Natural Alfredo Dugés de la Universidad de Guanajuato.

\section{La temática}

La propuesta metodológica que se presentará a continuación se orienta al público infantil, debido a que la temática ambiental requiere de refuerzo del conocimiento del entorno, así como del sistema valoral. 
Actualmente es preocupante la degradación ambiental que enfrenta el planeta, el desarrollo industrial, los residuos sólidos, y la sobre explotación de los recursos naturales ha traído consecuencias desastrosas para los ecosistemas, calidad del aire, contaminación del suelo, etc. Desde comienzos de los setenta han existido movimientos sociales y conferencias mundiales ha sido la concientización global acerca de la contaminación del medio ambiente, así como de diseñar estrategias revertir los daos y conservar los recursos naturales. Es aquí donde la educación tiene una importancia fundamental, ya que los valores son una necesidad para cambiar hábitos y actitudes en el ser humano.

La educación ambiental, por tanto, es el tema principal de esta propuesta metodológica teniendo al museo como espacio de divulgación y el aprovechamiento de sus recursos didácticos orientados a este fin.

\section{El museo}

El museo cuenta con recursos visuales que son importantes para vincular el contenido abstracto con el concreto. La exposición permanente ofrece múltiples posibilidades de sensibilización e interacción con el público visitante o el usuario del museo. En este ámbito se brindan grandes oportunidades para ofrecer al público narraciones y discursos integradores donde distintos grupos sociales y étnicos; grupos de género y también de edad pueden y deben estar presentes. (Peraile, Ruiz., \& Torreira, 2014).

En las exposiciones, se utilizan recursos de comunicación como: textos, ilustraciones, de carácter explicativo, documental, evocativo; fotografías, animaciones; diagramas, mapas, planos, cronologías, y otro tipo de elementos gráficos; esculturas, modelos, maquetas y otros recursos en tres dimensiones; así como nuevas tecnologías más o menos interactivas y/o multimedia, entre otros. Todos los recursos de la exposición, en su espacio arquitectónico y museográfico -accesos, recorridos, forma de salas, acabados en suelo, paredes y techos, texturas, colores, iluminación, confort y ambientación general- propician una determinada experiencia en la visita. (Peraile, et al., 2014).
En el público infantil, la percepción del espacio es diferente, es decir ciertos aspectos del diseño del espacio toman vital importancia como la escala, altura, colores contrastantes, imágenes visuales, etc. Todos estos elementos brindan en conjunto refuerzo del aprendizaje y comprensión de los contenidos durante la visita guiada, causando que la vista pueda ser contemplativa y a la vez agradable.

Las actividades didácticas y los talleres, son más efectivos cuando se brindan a los usuarios al término de la visita, ya que los visitantes están contextualizados y sensibilizados en la temática y por consiguiente es factible la reflexión y cambio social. Es primordial enfatizar que, en el sector infantil, el aprendizaje se estimula a través del juego, siendo la curiosidad lo que conlleva al descubrimiento; se debe por tanto potenciar las capacidades de exploración e imaginación. Al respecto Cabral \& Maldonado (2019) comentan

Para el público que visita estos espacios científicos, se busca brindarle un lugar de participación y encuentro con la ciencia, un ambiente de reflexión provocando el desarrollo del intelecto a través del juego y la experimentación en historia, tecnología, ciencia, astronomía, biología, geología, arte, entre otras áreas que se pueden combinar, dependiendo del enfoque que se pretenda dar, para que puedan propiciar al público el descubrir hechos desconocidos o poco entendidos de nuestro planeta. (p.4)

La propuesta se centra en el museo de Historia Natural Alfredo Dugés de la Universidad de Guanajuato como caso de estudio. Este museo cuenta con un acervo extenso de mamíferos, reptiles y aves, así como de especies endémicas e incluso extintas.

El museo ofrece una experiencia con especímenes reales disecados, los cuales permite potencializar la curiosidad, y el misterio del conocimiento de la evolución; así como conocer en el plano físico especies extintas que dan valor social y cultural a las cuestiones actitudinales y comportamentales en la vida cotidiana.

La museografía actual estuvo a cargo del Dr. Oscar J. Polaco Ramos en 2009, y está compuesta de varias salas de exposición: 
Sala 1, El Gabinete de Historia Natural, es una remembranza de lo que fue el antiguo gabinete de historia natural del Colegio del Estado. En ella se encuentra un foto-mural con la imagen del Dr. Alfredo Dugès, el preparador Vicente Fernández y sus alumnos.

Sala 2, Biodiversidad, tiene representado un ambiente marino del lado izquierdo, en el que se observan desde los corales, caracoles y equinodermos (estrellas, galletas y erizos de mar), hasta los tiburones y finalmente, un grupo diverso, el de las tortugas, donde observamos una gran variedad de colores, tamaños y formas. En la vitrina de lado derecho se representa un ambiente terrestre, en el que se muestran numerosas especies de mamíferos como ratones, conejos y murciélagos entre otros. Se aprecian varios pericos, tucanes, búhos, garzas y un flamenco.

Sala 3, Evolución, exhibe ejemplares que representan algunos de las múltiples disciplinas necesarias para el estudio de los procesos evolutivos a partir de la Biología Comparada. Se muestran algunos fósiles, que en conjunto con la sucesión de ellos en el tiempo geológico son usados como evidencia de que la evolución es un proceso que realmente ocurre.

Sala 4, Extinciones, es un pequeño espacio que alberga ejemplares de especies que se consideran extintas o que en la actualidad es raro encontrarlas en forma silvestre. Son especies que se enlistan en alguna de las categorías de riesgo establecidas ya sea por instituciones extranjeras o nacionales.

Sala 5, Guanajuato, se exhiben animales y plantas que viven en alguna parte del estado de Guanajuato. Este estado tiene una historia de más de 400 años de ocupación humana.

El acervo científico se almacena en un espacio cerrado con acceso restringido. Este acervo fue recientemente rescatado, revisado y curado. La colección cuenta con más de 6000 ejemplares biológicos catalogados.

La colección paleontológica cuenta con ejemplares de vertebrados e invertebrados y ha sido curada y registrada ante el Instituto Nacional de Antropología e Historia de México.

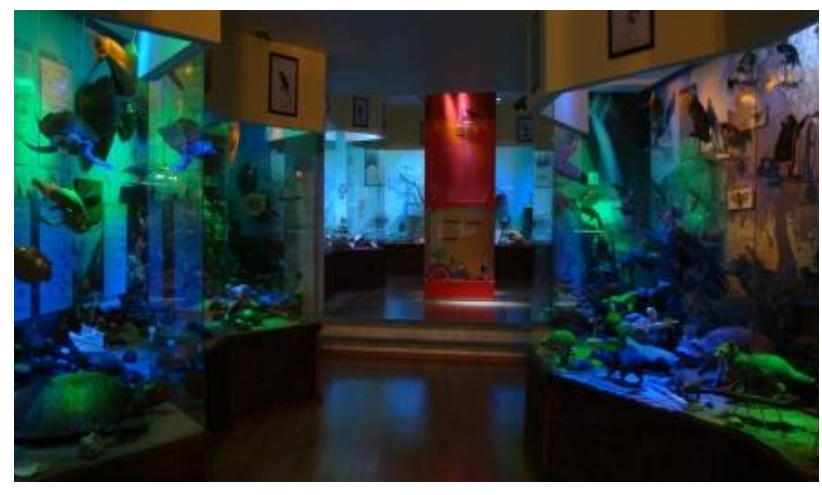

Figura 1 Museo de Historia Natural Alfredo Duges, y Archivo Museo, 2009

El museo por tanto provee un acervo que es congruente a la temática ambiental y permite conocer especies lo que constituye para el niño una oportunidad de vincular el conocimiento con la realidad tangible.

\section{Resultados}

El museo es un espacio ideal para el desarrollo de una propuesta metodológica en el área de educación ambiental, ya que la exposición permanente es congruente con los contenidos de educación ambiental y preservación de las especies.

El diseño de la propuesta está sustentado en tres ejes primordiales para la educación ambiental:

\section{- Contextualización \\ - Sensibilización \\ - Acción}

Las vertientes educativas de la propuesta corresponden al constructivismo social y el modelo educativo por competencias. Lo anterior, derivado de la necesidad de que el estudiante sea el promotor de su aprendizaje, ya que se encuentra en un espacio de educación informal en el cuál cada individuo construye su conocimiento en base a la experiencia y observación.

Otro aspecto que es tomado en cuenta para el diseño del modelo es la integración del docente como agente de conocimiento en el museo. La formación de profesores en educación no formal representa una alternativa para establecer relaciones, interactuar socialmente, conocer cómo aprende el niño fuera del espacio convencional educativo, desarrollar la conciencia y los valores. 
Vendrasco et al. (2018) señala "Cabe destacar que la formación de profesores en educación no formal debe ser un proceso reflexivo, por lo que no basta vivir la experiencia y no pensar sobre ella. Además, la formación de profesores en educación no formal requiere una planificación de parte del docente: es una práctica pedagógica intencionada". (p. 3) Las fases de la propuesta metodológica son las siguientes:

$1^{\text {a }}$ Fase: Actividad de divulgación del conocimiento. En esta fase, en la institución educativa se lleva a cabo una conferencia sobre la degradación ambiental con apoyo audiovisual. Es importante la contextualización no únicamente de forma global sino también regional, con la finalidad de que el estudiante relacione los contenidos con su entorno real y cotidiano.

El diseño de actividades complementarias es ideal para el refuerzo del conocimiento, para lo cual se integra la investigación documental electrónica sobre los tópicos de especies extintas y en peligro de extinción en México.

$2^{\mathrm{a}}$ Fase: Visita al Museo. En esta fase la visita se realiza al museo, procurando tener los materiales necesarios para llevar a cabo las actividades. Es fundamental que se realice una encuesta de entrada para conocer las expectativas de los educandos, que piensan del museo y cuáles han sido sus experiencias anteriores. La planificación de las actividades y talleres, así como la profundidad del aprendizaje dependerá del nivel educativo. Esta fase se divide en tres secciones:

\begin{tabular}{|c|c|c|}
\hline Observación & Experimentación & $\begin{array}{l}\text { Actividad de } \\
\text { divulgación }\end{array}$ \\
\hline $\begin{array}{lr}\text { Visita guiada } \\
\text { contribuye } & \text { al } \\
\text { conocimiento } & \\
\text { sobre evolución, } \\
\text { extinción, } & \text { y } \\
\text { especies en } & \text { el } \\
\text { Estado } & \\
\text { Guanajuato. } & \\
\end{array}$ & $\begin{array}{l}\text { Talleres sobre } \\
\text { educación } \\
\text { ambiental: } \\
\text { Los murciélagos } \\
\text { tus amigos } \\
\text { Mamíferos } \\
\text { Contaminación } \\
\text { ambiental }\end{array}$ & $\begin{array}{l}\text { Realización } \\
\text { de comic } \\
\text { científico } \\
\text { ambiental }\end{array}$ \\
\hline
\end{tabular}

Tabla 1 Modelo metodológico

Fuente: Elaboración Propia (2017)

$3^{\mathrm{a}}$ Fase: Dinámica de cierre y encuesta de salida, lo cuál permitirá conocer cambios de percepción en los visitantes. $4^{\mathrm{a}}$ Fase: Fase: Sesión de cierre en la institución educativa llevando a cabo la actividad: Reporteros ambientales, la cual consiste en la realización de un video que exponga una problemática ambiental, así como la solución colectiva.

$5^{\text {a }}$ Fase: Exposición del comic científico ambiental y de la divulgación de los videos a través de las redes sociales del Museo.

\section{Agradecimiento}

Departamento de Estudios Organizacionales de la División de Ciencias Económico Administrativas de la Universidad de Guanajuato.

\section{Conclusiones}

En México aún falta potencializar los recursos museísticos y establecer una relación más estrecha con las instituciones de educación; es decir, ir más allá de las visitas convencionales y convertir la experiencia en un medio de refuerzo del conocimiento, así como una oportunidad de aplicarlo.

El museo ha evolucionado de ser un espacio que almacena bienes valiosos a ser una alternativa educativa debido a la riqueza de sus recursos y las exposiciones. En este sentido, el museo de historia natural provee conocimientos y actividades que refuerzan el conocimiento del medio ambiente, evolución y la importancia de la preservación de los seres vivos.

El presente trabajo muestra la relación entre el museo, la educación informal y la educación ambiental; sin embargo, es necesario que se vinculen los conocimientos que se adquiere en el aula con los contenidos de las exposiciones y actividades de divulgación que se ofrece en el museo para que tenga impacto en el visitante y le permita reflexionar y construir nuevos saberes.

El público infantil requiere de estrategias que involucren actividades lúdicas y que incrementen la curiosidad y la experimentación. Lo anterior, supone un reto para los proyectos de divulgación ya que se debe tomar en cuenta la edad, nivel educativo, aspectos sociológicos y de comunicación para lograr establecer recursos didácticos que tengan impacto y conviertan la visita al museo en una experiencia de aprendizaje significativo.

SÁNCHEZ-RAMOS, Ma. Eugenia \& ZÁRATE-NEGRETE, Laura Elena. Modelo metodológico para la educación ambiental a partir del museo de ciencias. Revista de Pedagogía Crítica. 2019 


\section{Referencias}

Álvarez Domínguez, P. (2009). Espacios educativos y museos de pedagogía, enseñanza y educación. Cuestiones pedagógicas, 19, 191 206. Recuperado de: https://idus.us.es/xmlui/bitstream/handle/11441 /12865/file_1.pdf?sequence

Cabral, R., \& Rivera, J. L. M. (2019). Museos de ciencias en México: Educación y concientización cultural. Naturaleza y Tecnología. Recuperado de:

http://quimica.ugto.mx/index.php/nyt/article/vie w/343

Fenichel, M., \& Schweingruber, H. A. (2010). Surrounded by Science: Learning Science in Informal Environments. National Academies Press.

Gaudiano, E. G. (2001). Otra lectura a la historia de la educación ambiental en América Latina y el Caribe. Desenvolvimento e Meio Ambiente, 3.

Guisasola, J., \& Morentin, M. (2007). ¿ Qué papel tienen las visitas escolares a los museos de Ciencias en el aprendizaje de las Ciencias? Una revisión de las investigaciones. Enseñanza de las Ciencias, 25(3), 401-414. Recuperado de:

https://ddd.uab.cat/pub/edlc/02124521v25n3/02 124521v25n3p401.pdf

https://s3.amazonaws.com/academia.edu.docum ents/40328018/Infancia_museologia_y_Arque ologia.pdf?response-content-

disposition=inline $\% 3 \mathrm{~B} \% 20$ filename $\% 3 \mathrm{DInfanc}$ ia_museologia_y_arqueologia._Refle.pdf\&X-

Amz-Algorithm=AWS4-HMAC-SHA256\&X-

Amz-

Credential=AKIAIWOWYYGZ2Y53UL3A\%2

F20190913\%2Fus-east-

1\%2Fs3\%2Faws4_request\&X-Amz-

Date $=20190913 \mathrm{T010836Z \& X-Amz-}$

Expires $=3600 \& X-A m z-$

SignedHeaders $=$ host $\&$ X-Amz-

Signature $=0 \mathrm{a} 81309 \mathrm{~b} 670 \mathrm{ac} 141 \mathrm{e} 05 \mathrm{e} 61 \mathrm{f} 70 \mathrm{bd} 458$

3e1321dad965b08a526c85fcee97ddd3e2

Hein, G. (1995). The Constructivist Museum. Journal for Education in Museums, (16), 1995 21-23.
Hernández, Y. M. (2013). Lugares de memoria: entre la tensión, la participación y la reflexión. Panorama, 7(13), 97-109. Recuperado de: https://journal.poligran.edu.co/index.php/panor ama/article/view/434

Melgar, M. F. (2019). i Todo me gustó! Los niños y sus percepciones de una actividad educativa en un museo. Educatio Siglo XXI, 37(1 Marzo), 167-194. Recuperado de: https://revistas.um.es/educatio/article/view/363 451

Omedes, A. (2005). Los museos de Ciencias Naturales, piezas clave para la conservación de la Biodiversidad. Quark, (35), 72-78.

Peraile, I. I., Ruiz, C. L., \& Torreira, L. P. (2014). Infancia, museología y arqueología. Reflexiones en torno a los museos arqueológicos y el público infantil. Archivo de Prehistoria Levantina, 30. Recuperado de:

Vendrasco, N., Navarrete, T., Olivares, R., Astroza, V., \& Fuente, R. (2018). 9A012 Enseñar Ciencias fuera del aula: una experiencia con profesores en formación. Tecné Episteme y Didaxis TED, 1-6. Recuperado de: https://revistas.pedagogica.edu.co/index.php/TE D/article/view/9253 